\title{
Large $\mathrm{HDL}_{2}$ combined with inflammatory factors as superior predictors for coronary artery disease than small $\mathrm{HDL}_{3}$
}

\author{
Xiaoying Wu ${ }^{1 \#}$, Zhijian $\mathrm{He}^{2 \#}$, Runlu Sun ${ }^{1}$, Xiangkun $\mathrm{Xie}^{1}$, Qingqun Chen ${ }^{3}$, Junjie Wang ${ }^{1}$, Jinlan Bao ${ }^{4}$, \\ Jingjing Huang ${ }^{1}$, Yuan Jiang ${ }^{1}$, Yuling Zhang ${ }^{1}$, Jingfeng Wang ${ }^{1}$
}

${ }^{1}$ Cardiovascular Medicine Department, Sun Yat-sen Memorial Hospital, Sun Yat-sen University, Guangzhou, China; ${ }^{2}$ Cardiovascular Medicine Department, The First Affiliated Hospital/School of Clinical Medicine, Guangdong Pharmaceutical University, Guangzhou, China; ${ }^{3}$ Cardiovascular Medicine Department, Qingyuan People's Hospital, Qingyuan, China; ${ }^{4}$ Comprehensive Department, Sun Yat-sen Memorial Hospital, Sun Yat-sen University, Guangzhou, China

Contributions: (I) Conception and design: J Wang, Y Zhang, X Wu, Z He, R Sun, X Xie; (II) Administrative support: None; (III) Provision of study materials or patients: None; (IV) Collection and assembly of data: X Wu, Z He, R Sun, X Xie, Q Chen, J Wang, J Bao, J Huang, Y Jiang; (V) Data analysis and interpretation: X Wu, Z He, R Sun, X Xie, Q Chen, J Wang, J Bao, J Huang, Y Jiang; (VI) Manuscript writing: All authors; (VII) Final approval of manuscript: All authors.

\#These authors contributed equally to this work.

Correspondence to: Jingfeng Wang; Yuling Zhang. Cardiovascular Medicine Department, Sun Yat-sen Memorial Hospital, Sun Yat-sen University, Guangzhou, China. Email: wjingf@mail.sysu.edu.cn; zhyul@mail.sysu.edu.cn.

Background: This study investigated whether combinations of high-density lipoprotein (HDL) subfractions and inflammatory markers would add value to coronary artery disease (CAD) prediction.

Methods: Non-CAD subjects $(\mathrm{n}=245)$ were stratified into low/moderate/high-Framingham risk (L/M/H-FR) groups and $180 \mathrm{CAD}$ patients were enrolled. Levels of HDL-C, $\mathrm{HDL}_{2}, \mathrm{HDL}_{3}$, monocyte chemoattractant protein-1 (MCP-1), and high-sensitivity C-reactive protein (hsCRP) were measured. Multivariable logistic models for CAD were estimated with a single parameter or all parameters together after adjustment for conventional risk factors (CRFs), and Z statistics, net reclassification improvement (NRI), and integrated discrimination improvement (IDI) were used to compare discrimination among different models.

Results: The results show that HDL-C, $\mathrm{HDL}_{2}$, and $\mathrm{HDL}_{3}$ gradually decreased, while MCP-1 and hsCRP gradually increased from $\mathrm{L} / \mathrm{M} / \mathrm{H}-\mathrm{FR}$ to the CAD group. When applying a single factor in the CRFsadjusted models, HDL-C (OR 0.011, 95\% CI, 0.002-0.071, $\mathrm{P}<0.05)$ and $\mathrm{HDL}_{2}(\mathrm{OR} 0.000072,95 \% \mathrm{CI}$, 0.000001-0.004, $\mathrm{P}<0.05)$, but not $\mathrm{HDL}_{3}$, were significantly related to CAD risk. Only $\mathrm{HDL}_{2}(\mathrm{OR} 0.000072$, 95\% CI, 0.000001-0.004, $\mathrm{P}<0.001)$ remained significant when applying all HDL parameters. In the model including all HDL and inflammatory parameters, $\mathrm{HDL}_{2}$ (OR 0.001, 95\% CI, 0.000027-0.051), MCP-1 (OR 1.066, 95\% CI, 1.039-1.094), and hsCRP (OR 1.130, 95\% CI, 1.041-1.227) showed significant differences (all $\mathrm{P}<0.05)$. This combined model showed improved discrimination over the models with a single factor $(\mathrm{P}<0.05)$ or all HDL parameters $(\mathrm{Z}=3.299$, NRI $=0.179$, IDI $=0.081, \mathrm{P}<0.001)$.

Conclusions: Large $\mathrm{HDL}_{2}$ is superior to small $\mathrm{HDL}_{3}$ in the inverse association with CAD. The combination of $\mathrm{HDL}_{2}, \mathrm{MCP}-1$, and hsCRP with CRFs provides an optimal prediction for CAD.

Keywords: Coronary artery disease (CAD); high-density lipoprotein subfractions (HDL subfractions); monocyte chemoattractant factor-1; high-sensitivity C-reactive protein (hsCRP); combined prediction

Submitted Jan 24, 2021. Accepted for publication Apr 15, 2021.

doi: $10.21037 /$ atm-21-948

View this article at: http://dx.doi.org/10.21037/atm-21-948 


\section{Introduction}

High-density lipoprotein-cholesterol (HDL-C) levels have been demonstrated to be inversely associated with coronary artery disease $(\mathrm{CAD})$ risk in numerous epidemiological studies, including the Framingham Heart study (1-3). However, several recent clinical trials have challenged the value of raising HDL-C pharmacologically $(4,5)$, and Mendelian randomization studies also do not support the causal role of HDL-C in CAD development (6). Instead of HDL "quantity", growing interest has focused on the value of HDL "quality", such as HDL subfractions and their differential atheroprotective functions (7).

HDL can be separated into two principal subfractions with ultracentrifugation: large buoyant $\mathrm{HDL}_{2}$ and small dense $\mathrm{HDL}_{3}$. Previous studies have suggested that HDL subfractions might add information in CAD risk assessment and Hirano et al. (8) developed a simpler and more precise method using a single-step precipitation to measure the levels of $\mathrm{HDL}_{2}$ and $\mathrm{HDL}_{3}$. $\mathrm{HDL}_{2}$ is often thought to be the "protective" form of HDL, and has been shown to have an inverse relationship with the risk of CAD in both crosssectional and prospective studies $(9,10)$. Our previous study has also consistently confirmed the inverse relationship of large $\mathrm{HDL}_{2}$ with premature CAD risk (11). However, conflicting results suggest that $\mathrm{HDL}_{3}$ is superior to $\mathrm{HDL}_{2}$ in predicting the incidence and mortality of ischaemic heart disease $(12,13)$, and it remains to be determined which of the two is the more powerful negative risk factor for CAD and whether HDL subfractions provide a better predictive value than $\mathrm{HDL}-\mathrm{C}$ itself.

Chronic persistent inflammation is another critical mechanism in the atherothrombotic process, and a substantial proportion of unexplained vascular disease might be related to inflammation (14). Monocyte chemoattractant factor-1 (MCP-1) is a chemoattractant that recruits monocytes to the subendothelial space and initiates plaque formation. Georgakis et al. (15) have shown that genetically determined higher MCP-1 levels are associated with a higher risk of CAD, myocardial infarction, and stroke, while the prospective PRIME study did not confirm these same associations (16). High-sensitivity C-reactive protein (hsCRP) is another inflammatory marker that displays a positive and independent relationship with cardiovascular morbidity and mortality $(17,18)$. However, adding hsCRP levels to existing CAD risk assessment models demonstrates only modest improvement (19). Thus, the roles of MCP-1 and hsCRP in CAD risk prediction still require further research.

Since 1998, the original Framingham risk scores (FRS) (20) have incorporated age, sex, diabetes, systolic and diastolic blood pressures, levels of total and HDL cholesterol, and smoking to estimate a model for predicting the 10-year coronary heart disease risk and guide therapy for primary prevention. Considering that the original FRS ignored the risk prediction of other common cardiovascular disease (CVD), such as stroke, an FRS was developed in 2008 for the prediction of CVD (21). However, these classic tools have been shown to overestimate cardiovascular events by $8 \%$ to $154 \%$ in the MESA trial (22), calling for a combination of new risk factors to improve its performance. Ridker et al. (23) revealed that the combined evaluation of both LDL-C and hsCRP was superior as a method of cardiovascular risk detection than the measurement of either biologic marker alone, but little is known about the combined value of HDL subfractions with inflammatory markers in CAD risk assessment.

Therefore, we conducted this cross-sectional study, which enrolled 245 non-CAD subjects with low/moderate/ high Framingham risk (L/M/H-FR) and $180 \mathrm{CAD}$ patients, and used the new single-step precipitation method (8) to measure $\mathrm{HDL}_{2}$ and $\mathrm{HDL}_{3}$ levels. We found both $\mathrm{HDL}_{2}$ and $\mathrm{HDL}_{3}$ gradually decreased from the L/M/H-FR group to the CAD group. Large $\mathrm{HDL}_{2}$ is superior to small $\mathrm{HDL}_{3}$ in the inverse association with CAD. Furthermore, the combination of $\mathrm{HDL}_{2}, \mathrm{MCP}-1$, and hsCRP with CRFs provides an optimal prediction for CAD risk. We present the following article in accordance with the MDAR checklist (available at http://dx.doi.org/10.21037/atm-21948).

\section{Methods}

\section{Study design and population}

The study complied with the Declaration of Helsinki (as revised in 2013) and was approved by the hospital's ethics review board (Sun Yat-sen Memorial Hospital, Guangzhou, China, IRB number SYSEC-KY-KS-2020-083-001). All subjects provided written informed consent to participate in the study. The study had been registered in Chinese Clinical Trial Registry as "A study on the predictive value of inflammatory markers and blood lipid profiles in the risk of coronary artery disease". The clinical trial registration number is ChiCTR2000038859.

From September to November 2020, 245 asymptomatic 
subjects with no known CAD were recruited from our physical examination centre. According to the Framingham risk score [1998] (20), these participants were stratified into three groups: low-Framingham risk (L-FR, 10 -year CAD risk $<10 \%, \mathrm{n}=120$ ), moderate-Framingham risk $(M-F R, 10 \% \leq 10$-year CAD risk $<20 \%, n=51)$ and high Framingham risk (H-FR, 10-year CAD risk $\geq 20 \%, n=74$ ), and all of these participants were defined as the Framingham risk group (FR group). Meanwhile, 180 patients with angiographically diagnosed CAD (at least $50 \%$ obstructive lesions of one or more coronary arteries) in our cardiovascular medicine department were enrolled as the CAD group.

Subjects were excluded if they were suffering from acute coronary syndrome, advanced congestive heart failure, arrhythmia, or any infectious or systematic inflammatory disease over the previous 1 month. Other exclusion criteria included subjects less than 18 years or over 80 years, pregnancy or lactation, the existence of structural heart disease or cardiomyopathy, severe liver or renal dysfunction, thyroid disease, significant haematologic disorders, malignancy, major surgery or severe trauma within 2 months, and a history of the use of lipid-lowering drugs, antioxidants, glucocorticoid, or nonsteroidal antiinflammatory drugs within the past 3 months. All exclusion criteria were preestablished.

Hypertension was defined as repeated BP measurements $\geq 140 / 90 \mathrm{mmHg}$ (at least two times on different days) or self-reported hypertension with current use of antihypertension drugs. Diabetes mellitus was defined as fasting plasma glucose $\geq 126 \mathrm{mg} / \mathrm{dL}(7.0 \mathrm{mmol} / \mathrm{L})$, selfreported diabetes, or use of anti-diabetic medications. A family history of premature CAD (PCAD) was defined as myocardial infarction occurrence before the age of 55 for men and 65 for women in first-degree relatives.

\section{Measurement of HDL-C subfractions and other lipid profiles}

Fasting blood samples were collected in EDTA tubes at baseline. After centrifugation at $4{ }^{\circ} \mathrm{C}$, all plasma aliquots were stored at $-80^{\circ} \mathrm{C}$ until analysis.

The levels of HDL-C were measured by homogeneous HDL-EX HDL-C assays (Denka Seiken, Tokyo, Japan) and HDL subfractions were analysed by a single-step precipitation method (8). The precipitation reagent $(0.06 \mathrm{~mL})$, containing heparin, $\mathrm{MnCl}_{2}$, and dextran sulfate, was added to $0.3 \mathrm{~mL}$ of serum, mixed, left at room temperature for $30 \mathrm{~min}$, and centrifuged at 10,000 rpm for $10 \mathrm{~min}$ at $4{ }^{\circ} \mathrm{C}$. Both the apolipoprotein $\mathrm{B}$ (apo B)containing lipoproteins and $\mathrm{HDL}_{2}$ could be simultaneously precipitated, and an aliquot of the supernatant was taken for $\mathrm{HDL}_{3}$ measurement using homogenous HDL-EX HDL-C assays. There were biological replicates for three times when testing the levels of HDL-C and $\mathrm{HDL}_{3}$. The measured value for total $\mathrm{HDL}_{3}$ was multiplied by 1.2 to correct for dilution by the reagents and levels of $\mathrm{HDL}_{2}$ were derived from the formula: $\mathrm{HDL}_{2}=\mathrm{HDL}-\mathrm{C}-\mathrm{HDL}_{3}$.

Other lipid profiles, including triglyceride (TG), total cholesterol (TC), low-density lipoprotein-cholesterol (LDL-C), and apolipoprotein A1 (apo A1) and apo B, were measured on the Hitachi Modular Analytics System (Roche Modular DPP; Hitachi Ltd, Tokyo, Japan). Non-HDL-C levels were calculated by subtracting HDL-C from TC.

\section{Measurement of inflammatory markers}

The levels of plasma MCP-1 were measured by ELISA (BMS281TEN, Austria) on an automated microplate reader (Multiskan MK3, Thermo Fisher Scientific, USA). High-sensitivity testing for C-reactive protein (CRP) was performed using an immunoturbidimetric assay (GS621M, China) on the Hitachi Modular Analytics System.

\section{Statistical analysis}

Continuous variables with a Gaussian distribution were reported as the means \pm standard deviations, and their differences between the FR group and CAD group were assessed by independent $t$-test. Continuous variables without a Gaussian distribution were reported as medians with interquartile ranges with differences compared by the Mann-Whitney U test. Categorical variables were reported as case numbers (proportions), and differences were compared using the Chi-squared test. Accordingly, the differences among the L/M/H-FR and CAD groups were compared by one-way ANOVA, the Kruskal-Wallis $\mathrm{H}$ test, or the Chi-squared test. The pairwise comparisons among the four groups were conducted by the Tukey or Game-Howell test for Gaussian-distributed variables and by the Kruskal-Wallis $\mathrm{H}$ test for others without a Gaussian distribution.

Multivariable logistic regression analysis was performed to estimate the CAD risk models. All models were adjusted for conventional risk factors (CRFs), including age, sex, body mass index (BMI), systolic blood pressure (SBP), 
Table 1 Baseline characteristics in the Framingham risk group and CAD group

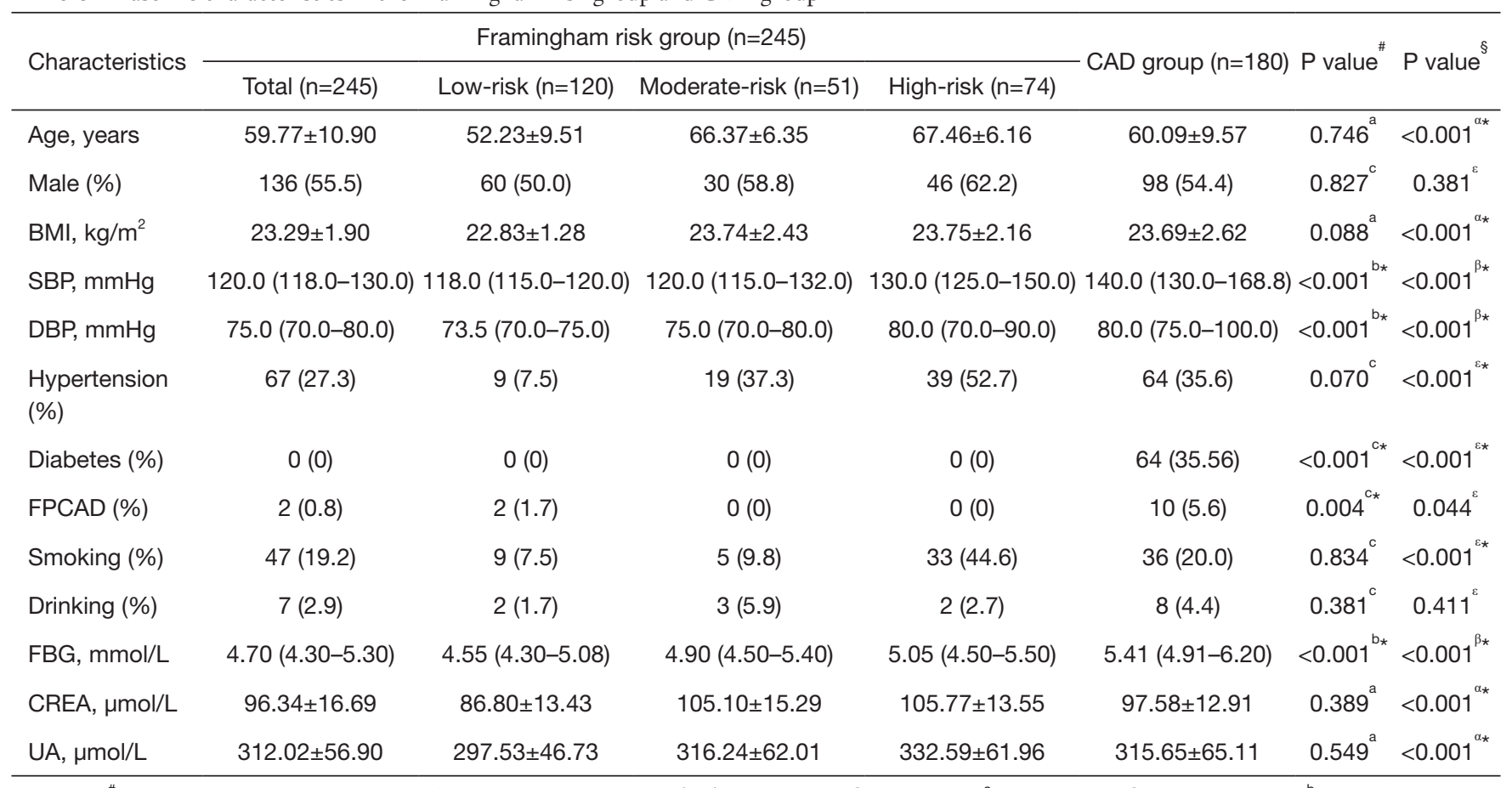

$\mathrm{P}_{\text {value }}{ }^{\#}$ represents the comparison of the Framingham risk (FR) group and CAD group; ${ }^{a}$, represents Student's $t$-test; ${ }^{b}$, represents the Mann-Whitney $\mathrm{U}$ test; ${ }^{\circ}$, represents the Chi-squared test. $\mathrm{P}$ value ${ }^{\S}$ represents the comparison of the low/moderate/high-FR group and CAD group; ${ }^{\alpha}$, represents one-way ANOVA; ${ }^{\beta}$, represents Kruskal-Wallis $\mathrm{H}$ test; ${ }^{\varepsilon}$, represents Chi-squared test; ${ }^{*}, \mathrm{P}<0.05$ as significance. CAD, coronary artery disease; BMI, body mass index; SBP, systolic blood pressure; DBP, diastolic blood pressure; FPCAD, family history of premature coronary artery disease; FBG, fasting blood glucose; CREA, creatinine; UA, uric acid.

diastolic blood pressure (DBP), hypertension, family history of PCAD, smoking, fasting blood glucose (FBG), creatinine (CREA), TG, TC or LDL-C, apo A1 and apo B, which were based on univariate analysis and general knowledge of CAD risk. Models 1 to 8 referred to the model applied with a single factor of HDL parameters (HDL-C, $\mathrm{HDL}_{2}$, $\mathrm{HDL}_{3}, \mathrm{HDL}_{2} / \mathrm{HDL}_{3}, \mathrm{HDL}_{2} / \mathrm{HDL}-\mathrm{C}$, and $\mathrm{HDL}_{3} / \mathrm{HDL}-\mathrm{C}$ ) or inflammatory markers (MCP-1 and hsCRP) sequentially. Model 9 was applied with all six HDL parameters, and model 10 incorporated all six HDL parameters and the two inflammatory markers together. Odds ratios (ORs) with 95\% CIs were calculated for these variables and shown in forest plots.

The discrimination of every model was assessed by the C-statistic, which was analogous to the area under the receiver operating characteristic (ROC) curve. The $\mathrm{Z}$ value, the index for the difference between two $\mathrm{C}$-statistics, was applied to compare discrimination between the two models. Reclassification improvement was defined as an increase in risk category for individuals who suffered events and as a decrease for those who did not (21), and both net reclassification improvement (NRI) and integrated discrimination improvement (IDI) were used for comparison of reclassification between two models. When calculating NRI, clinically based cut-off points of $10 \%$ and $20 \%$ were used (21).

All statistical analyses were performed by SPSS software (version 25.0, SPSS, Chicago, Illinois, USA) and R version 3.6.2 (R Foundation for Statistical Computing, Vienna, Austria). A two-tailed $\mathrm{P}<0.05$ was considered statistically significant.

\section{Results}

\section{Baseline characteristics}

The baseline demographic and clinical characteristics of the FR and CAD groups are shown in Table 1.

Participants in both FR and CAD groups were mainly middle-aged to elderly, and slightly over half were men. In comparison with the FR group, patients in the CAD group had higher SBP and DBP, a higher percentage of diabetes, 


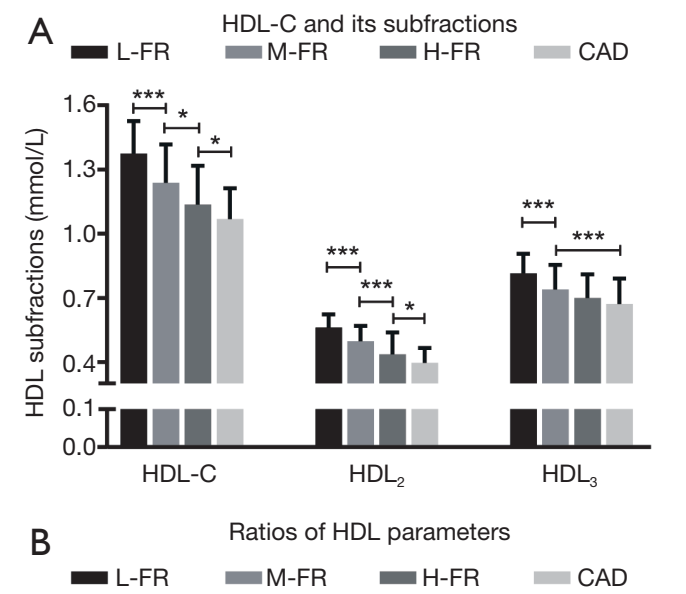

C $\quad \mathrm{MCP}^{\mathrm{N}}$
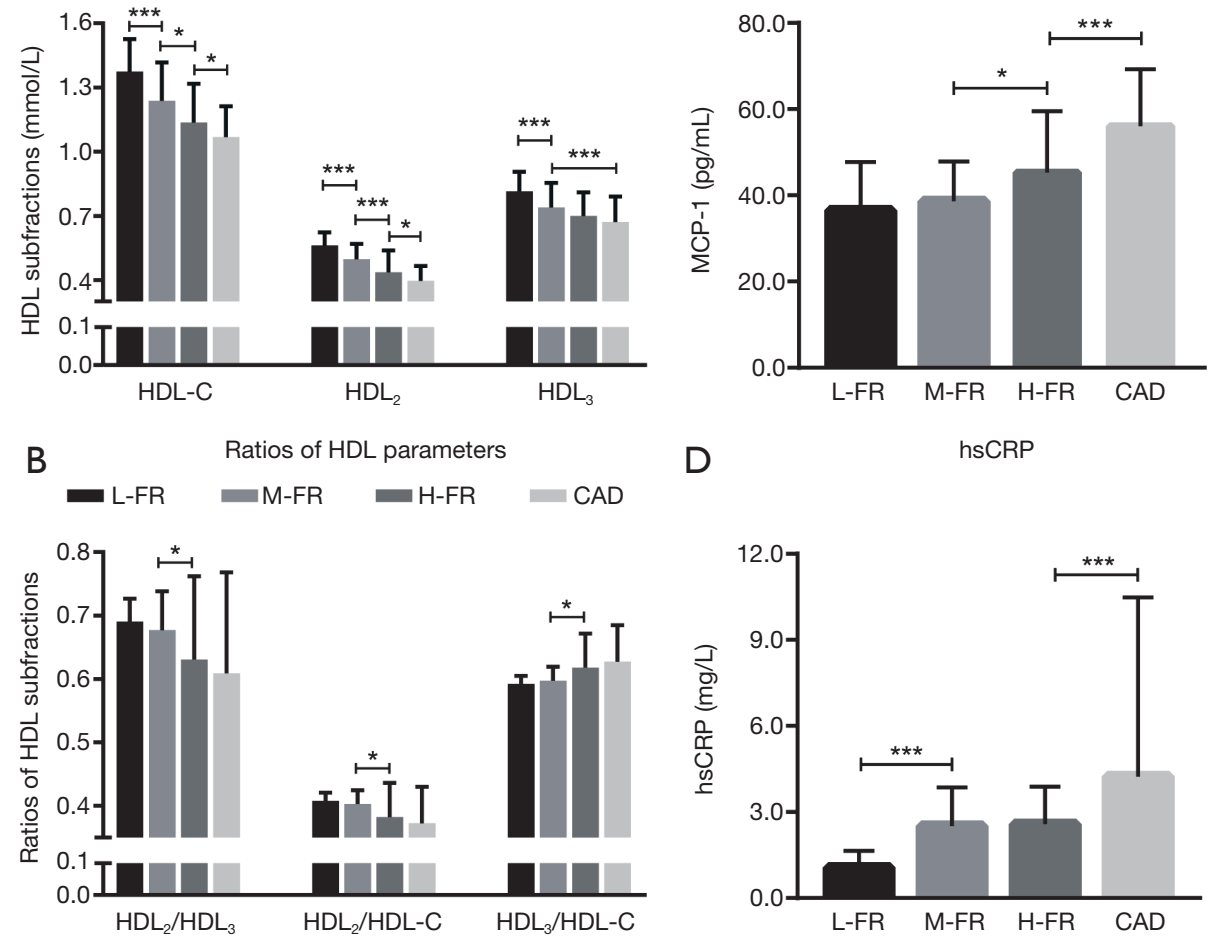

Figure 1 Pairwise comparisons of HDL parameters and inflammatory markers between the Low/Moderate/High Framingham risk group and the CAD group. (A) HDL-C and its subfraction concentrations; (B) ratios of HDL parameters; (C) MCP-1 concentration; (D) hsCRP concentration. ${ }^{*} \mathrm{P}<0.05$; ${ }^{* *} \mathrm{P}<0.001$. $\mathrm{P}<0.05$ was considered significant. HDL, high-density lipoprotein; CAD, coronary artery disease; MCP-1, monocyte chemoattractant protein-1; hsCRP, high-sensitivity C-reactive protein.

and positive family history of PCAD. FBG was higher in the CAD group, whereas CREA and uric acid (UA) did not show significant differences between the two groups. Furthermore, compared with the Low- and Moderate-FR groups, the High-FR group was more likely to have older subjects, more males, subjects with higher SBP levels, and a higher percentage of hypertension, diabetes, and smoking status.

\section{Analysis of HDL-C subfractions}

Table 1 shows $\mathrm{HDL}_{2}$ accounted for approximately twofifths of HDL-C, and $\mathrm{HDL}_{3}$ accounted for the other threefifths. All HDL-C, $\mathrm{HDL}_{2}$, and $\mathrm{HDL}_{3}$ levels were lower in the CAD group than in the FR group (HDL-C: $1.07 \pm 0.14$ vs. $1.27 \pm 0.20 ; \mathrm{HDL}_{2}$ : $0.40 \pm 0.07$ vs. $0.51 \pm 0.09 ; \mathrm{HDL}_{3}$ : $0.67 \pm 0.12$ vs. $0.76 \pm 0.11$; all $\mathrm{P}<0.001$, respectively).

The distributions of both $\mathrm{HDL}_{2}$ and $\mathrm{HDL}_{3}$ were consistent with HDL-C, with a reduced level across the
L/M/H-FR and CAD groups (HDL-C: $1.38 \pm 0.15$ vs. $1.24 \pm 0.18$ vs. $1.14 \pm 0.18$ vs. $1.07 \pm 0.14$; $\mathrm{HDL}_{2}: 0.56 \pm 0.06$ vs. $0.50 \pm 0.07$ vs. $0.44 \pm 0.10$ vs. $0.40 \pm 0.07 ; \mathrm{HDL}_{3}: 0.81 \pm 0.09$ vs. $0.74 \pm 0.11$ vs. $0.70 \pm 0.11$ vs. $0.67 \pm 0.12$; all $\mathrm{P}<0.001$, respectively). However, only $\mathrm{HDL}-\mathrm{C}$ and $\mathrm{HDL}_{2}$, but not $\mathrm{HDL}_{3}, \mathrm{HDL}_{2} / \mathrm{HDL}_{3}, \mathrm{HDL}_{2} / \mathrm{HDL}-\mathrm{C}$, or $\mathrm{HDL}_{3} / \mathrm{HDL}-\mathrm{C}$ showed significant differences in pairwise comparisons among all four groups (Figure 1A,B).

\section{Analysis of inflammatory markers}

As shown in Table 2, both MCP-1 and hsCRP levels were significantly higher in the CAD group than in the FR group [MCP-1: $56.09 \pm 13.16$ vs. $39.60 \pm 12.45$; hsCRP: 4.24 (2.56-10.48) vs. 1.53 (0.77-2.84); $\mathrm{P}<0.001$, respectively] and also showed a gradual increase from the L/M/H-FR group to the CAD group. The difference of MCP-1 was not significant between the L-FR and M-FR groups but was significant in pairwise comparisons among the M-FR, 
Table 2 Biochemistry parameters of lipid profiles and inflammation in the Framingham risk group and CAD group

\begin{tabular}{|c|c|c|c|c|c|c|c|}
\hline Variable & \multicolumn{4}{|c|}{ Framingham risk group $(n=245)$} & CAD group $(n=180)$ & $\mathrm{P}$ value & $P$ value ${ }^{\S}$ \\
\hline \multicolumn{8}{|l|}{ HDL parameters } \\
\hline $\mathrm{HDL}-\mathrm{C}, \mathrm{mmol} / \mathrm{L}$ & $1.27 \pm 0.20$ & $1.38 \pm 0.15$ & $1.24 \pm 0.18$ & $1.14 \pm 0.18$ & $1.07 \pm 0.14$ & $<0.001^{a \star}$ & $<0.001^{\alpha \star}$ \\
\hline $\mathrm{HDL}_{2}, \mathrm{mmol} / \mathrm{L}$ & $0.51 \pm 0.09$ & $0.56 \pm 0.06$ & $0.50 \pm 0.07$ & $0.44 \pm 0.10$ & $0.40 \pm 0.07$ & $<0.001^{a *}$ & $<0.001^{\alpha *}$ \\
\hline $\mathrm{HDL}_{2} / \mathrm{HDL}_{3}$ & $0.67 \pm 0.09$ & $0.69 \pm 0.04$ & $0.68 \pm 0.06$ & $0.63 \pm 0.13$ & $0.61 \pm 0.16$ & $<0.001^{a *}$ & $<0.001^{\alpha_{*}}$ \\
\hline $\mathrm{HDL}_{2} / \mathrm{HDL}-\mathrm{C}$ & $0.40 \pm 0.03$ & $0.41 \pm 0.01$ & $0.40 \pm 0.02$ & $0.38 \pm 0.05$ & $0.37 \pm 0.06$ & $<0.001^{a *}$ & $<0.001^{\alpha_{*}}$ \\
\hline $\mathrm{HDL}_{3} / \mathrm{HDL}-\mathrm{C}$ & $0.60 \pm 0.03$ & $0.59 \pm 0.01$ & $0.60 \pm 0.02$ & $0.62 \pm 0.05$ & $0.63 \pm 0.06$ & $<0.001^{a_{*}}$ & $<0.001^{a *}$ \\
\hline \multicolumn{8}{|l|}{ Standard lipid profiles } \\
\hline LDL-C, mmol/L & $2.93 \pm 0.97$ & $2.25 \pm 0.49$ & $3.01 \pm 0.59$ & $3.96 \pm 0.83$ & $3.87 \pm 0.96$ & $<0.001^{a_{*}}$ & $<0.001^{\alpha *}$ \\
\hline Non-HDL-C, mmol/L & $3.45 \pm 1.08$ & $2.68 \pm 0.55$ & $3.56 \pm 0.56$ & $4.61 \pm 0.92$ & $4.29 \pm 1.03$ & $<0.001^{a *}$ & $<0.001^{\alpha *}$ \\
\hline apo $A 1, g / L$ & $1.30 \pm 0.17$ & $1.39 \pm 0.15$ & $1.24 \pm 0.12$ & $1.19 \pm 0.14$ & $1.14 \pm 0.14$ & $<0.001^{a *}$ & $<0.001^{\alpha *}$ \\
\hline apo B, g/L & $0.91 \pm 0.12$ & $0.88 \pm 0.12$ & $0.93 \pm 0.10$ & $0.95 \pm 0.12$ & $1.04 \pm 0.69$ & $0.013^{a_{\star}}$ & $<0.001^{\alpha *}$ \\
\hline \multicolumn{8}{|l|}{ Inflammatory markers } \\
\hline $\mathrm{MCP}-1, \mathrm{pg} / \mathrm{mL}$ & $39.60 \pm 12.45$ & $36.51 \pm 11.28$ & $38.64 \pm 9.18$ & $45.26 \pm 14.29$ & $56.09 \pm 13.16$ & $<0.001^{a *}$ & $<0.001^{\alpha *}$ \\
\hline hsCRP, mg/L & $1.53(0.77-2.84)$ & $1.07(0.65-1.65)$ & $2.51(0.96-3.86)$ & $2.58(1.28-3.89)$ & $4.24(2.56-10.48)$ & $<0.001^{b_{*}}$ & $<0.001^{\beta_{*}}$ \\
\hline
\end{tabular}

$\mathrm{P}$ value ${ }^{\#}$ represents the comparison of the Framingham risk (FR) group and CAD group; ${ }^{a}$, represents Student's $t$-test; ${ }^{\mathrm{b}}$, represents the Mann-Whitney $\mathrm{U}$ test. $\mathrm{P}$ value ${ }^{\S}$ represents the comparison of the low/moderate/high-FR group and CAD group; ${ }^{\alpha}$, represents one-way ANOVA; ${ }^{\beta}$, represents the Kruskal-Wallis $\mathrm{H}$ test; ${ }^{*}, \mathrm{P}<0.05$ as significance. HDL-C, high-density lipoprotein-cholesterol; TG, triglyceride; TC, total cholesterol; LDL-C, low-density lipoprotein-cholesterol; apo A1, apolipoprotein A1; apo B, apolipoprotein B; MCP-1, monocyte chemoattractant protein-1; hsCRP, high-sensitivity C-reactive protein.

$\mathrm{H}-\mathrm{FR}$, and CAD groups (all $\mathrm{P}<0.05$, Figure 1C). The level of hsCRP was significantly different in the comparisons between the L-FR and M-FR groups as well as between the H-FR and CAD groups $(\mathrm{P}<0.001$, respectively $)$ but was similar between the M-FR and $\mathrm{H}-\mathrm{FR}$ groups $(\mathrm{P}>0.05)$ (Figure 1D).

\section{Multivariable logistic regression analysis}

In models 1 to 8 , which included a single parameter sequentially after adjustment for CRFs (Figure 2), both HDL-C (OR 0.011, 95\% CI, 0.002-0.071; $\mathrm{P}<0.001)$ and $\mathrm{HDL}_{2}$ (OR 0.000072, 95\% CI, 0.000001-0.004; $\mathrm{P}<0.001$ ) were independently associated with CAD risk in models 1 and 2, while $\mathrm{HDL}_{3}$ and three ratios of HDL parameters failed to be significantly related to CAD. In addition to inflammatory markers, MCP-1 (OR 1.082, 95\% CI,
1.056-1.108; $\mathrm{P}<0.001)$ and hsCRP (OR 1.160, 95\% CI, 1.068-1.260; $\mathrm{P}<0.001)$ demonstrated positive relationships with CAD risk in models 7 and 8.

Further, when applying all six HDL parameters in model 9, only large $\mathrm{HDL}_{2}$ (OR 0.000072, 95\% CI, 0.0000010.004; $\mathrm{P}<0.001)$, rather than HDL-C or small $\mathrm{HDL}_{3}$, maintained the inverse relationship with $\mathrm{CAD}$ (Figure 2).

Finally, when all six HDL parameters and two inflammatory markers were combined into model $10, \mathrm{HDL}_{2}$ (OR 0.001, 95\% CI, 0.000027-0.051; $\mathrm{P}<0.001$ ), MCP-1 (OR 1.066, 95\% CI, 1.039-1.094; $\mathrm{P}<0.001$ ) and hsCRP (OR $1.130,95 \% \mathrm{CI}, 1.041-1.227 ; \mathrm{P}=0.003)$ showed significance (Figure 2).

\section{Model fit and risk reclassification}

Models 1, 2, and 7 to 10 were defined as significant 


$\begin{array}{cl}\text { Model } & \text { Parameter } \\ 1^{\mathrm{a}} & \mathrm{HDL}-\mathrm{C} \\ 2^{\mathrm{a}} & \mathrm{HDL}_{2} \\ 3^{\mathrm{a}} & \mathrm{HDL}_{3} \\ 4^{\mathrm{a}} & \mathrm{HDL}_{2} / \mathrm{HDL}_{3} \\ 5^{\mathrm{a}} & \mathrm{HDL}_{2} / \mathrm{HDL}-\mathrm{C} \\ 6^{\mathrm{a}} & \mathrm{HDL}_{3} / \mathrm{HDL}-\mathrm{C} \\ 7^{\mathrm{a}} & \mathrm{MCP}-1 \\ 8^{\mathrm{a}} & \mathrm{hSCRP} \\ 9^{\mathrm{b}} & \mathrm{HDL}_{2} \\ 1^{\mathrm{c}} & \mathrm{HDL}_{2} \\ & \mathrm{MCP}-1 \\ & \text { hsCRP }\end{array}$
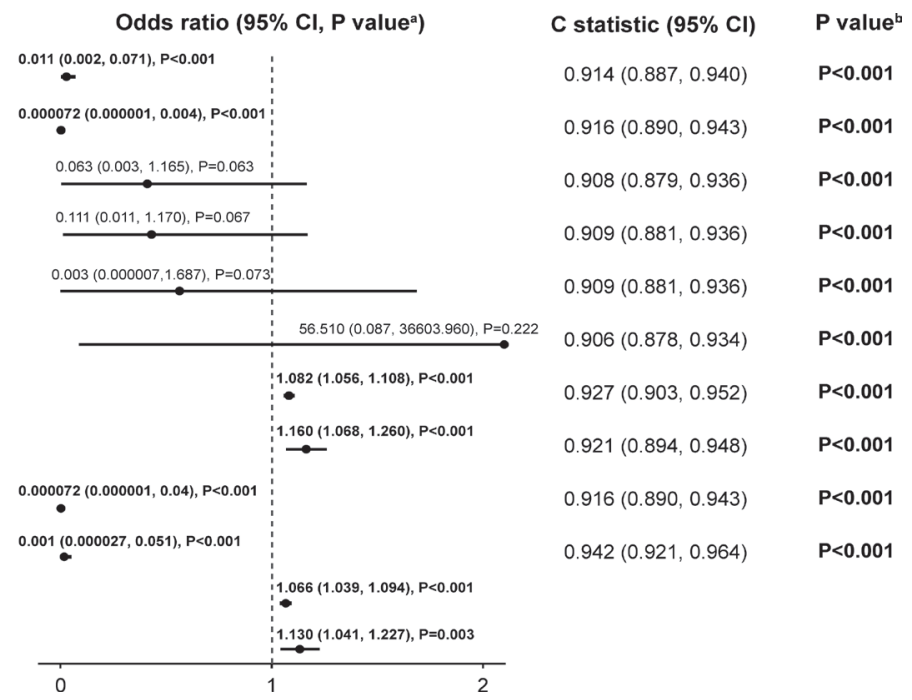

Figure 2 CAD risk prediction models by HDL subfractions and/or inflammatory markers. Multivariable logistic regression analysis was performed to evaluate the associations of HDL subfractions and/or inflammatory markers with CAD risk. Odds ratios (ORs) were estimated with $95 \%$ confidence intervals (CIs); $\mathrm{P}$ value ${ }^{a}$ represent the significance of ORs. The C-statistics of every model were computed; the $\mathrm{P}$ value ${ }^{\mathrm{b}}$ represents the significance of the $\mathrm{C}$-statistics with $\mathrm{P}<0.05$ in bold type as significance. ${ }^{\text {a }}$, refers to a model including a single $\mathrm{HDL}$ (HDL-C, $\mathrm{HDL}_{2}, \mathrm{HDL}_{3}, \mathrm{HDL}_{2} / \mathrm{HDL}_{3}, \mathrm{HDL}_{2} / \mathrm{HDL}-\mathrm{C}$ or $\mathrm{HDL}_{3} / \mathrm{HDL}-\mathrm{C}$ ) or inflammatory (MCP-1 or hsCRP) parameter after adjusting for conventional risk factors (CRFs). ${ }^{\text {, }}$, refers to a model including all six HDL parameters together after adjusting for CRFs. ${ }^{\mathrm{c}}$, refers to a model including all six HDL parameters and two inflammatory markers after adjusting for CRFs. HDL, high-density lipoprotein; CAD, coronary artery disease; MCP-1, monocyte chemoattractant protein-1; hsCRP, high-sensitivity C-reactive protein; CRF, conventional risk factor.

models with significant ORs of HDL parameters and/ or inflammatory markers $(\mathrm{P}<0.05)$, and their fits were compared by $Z$ value, NRI, and IDI.

As shown in Figure 2, model 2 with $\mathrm{HDL}_{2}$ had a higher C-statistic than model 1 with HDL-C (0.916 vs. 0.914). However, the discrimination of model 2 was virtually identical to that of model 1 with a nonsignificant $\mathrm{Z}$ value $(0.595, \mathrm{P}=0.552$, Figure $3 A$ ), and no reclassification improvement was observed with a negative NRI (0.011, $\mathrm{P}=0.731$ ) and IDI $(0.014, \mathrm{P}=0.078)$ (Figure $3 A, B)$.

Model 10 was applied with significant $\mathrm{HDL}_{2}, \mathrm{MCP}-$ 1 , and hsCRP. Compared with the four significant models (models 1, 2, 7, and 8) that included a single factor sequentially, model 10 showed a significantly higher C-statistic (0.942 vs. 0.914, 0.916, 0.927 and 0.921, all $\mathrm{P}<0.05$ ), respectively (Figures $2,3 A, C, D, E, F$ ) and an improvement in reclassification with positive NRI and IDI (NRI: 0.203, 0.179, 0.105, 0.172, respectively; IDI: 0.095, 0.081, 0.049, 0.052, respectively; all $\mathrm{P}<0.05$ ) (Figure $3 A$ ). Furthermore, in the comparison with model 9 incorporating all six HDL parameters, model 10 also showed improved fit on all measures with a $Z$ value of $3.299(\mathrm{P}<0.001)$, NRI of
$0.179(\mathrm{P}<0.001)$, and IDI of $0.081(\mathrm{P}<0.001)$ (Figure $3 A, G)$.

\section{Discussion}

In the present study, we found that the levels of HDL-C, $\mathrm{HDL}_{2}$, and $\mathrm{HDL}_{3}$ gradually decreased while MCP-1 and hsCRP gradually increased from the L/M/H-FR group to the CAD group. Both HDL-C and large $\mathrm{HDL}_{2}$, but not small $\mathrm{HDL}_{3}$ or the ratios of HDL parameters, showed an inverse and independent association with CAD risk. Only $\mathrm{HDL}_{2}$ remained significant in the model including all six HDL parameters. Moreover, the combined model with $\mathrm{HDL}_{2}, \mathrm{MCP}-1$, and hsCRP had better discrimination and risk reclassification than the models including either a single factor or all HDL parameters.

HDLs comprise a family of heterogeneous particles that vary by size, density, composition, and functionality. Two distinct HDL subfractions, large buoyant $\mathrm{HDL}_{2}$ and small dense $\mathrm{HDL}_{3}$, might exert differential effects on atherosclerosis and display a promising role in CAD risk prediction. In our study, large $\mathrm{HDL}_{2}$ levels, but not small $\mathrm{HDL}_{3}$ or three ratios of HDL parameters, showed 


\begin{tabular}{cccccccc}
\hline A Model comparison & $\begin{array}{c}\text { Change in } \\
\text { C-statistic }\end{array}$ & Z value & P value $^{\mathrm{a}}$ & $\begin{array}{c}\text { NRI } \\
(\mathbf{9 5 \%} \mathrm{Cl})\end{array}$ & P value $^{\mathrm{b}}$ & $\begin{array}{c}\text { IDI } \\
(\mathbf{9 5} \% \mathrm{Cl})\end{array}$ & P value $^{\mathrm{c}}$ \\
\hline model 2 vs model 1 & 0.002 & 0.595 & 0.552 & $0.011(-0.052,0.075)$ & 0.731 & $0.014(-0.002,0.029)$ & 0.078 \\
model 10 vs model 1 & 0.028 & 3.186 & $\mathbf{0 . 0 0 1}$ & $0.203(0.123,0.284)$ & $<0.001$ & $0.095(0.063,0.127)$ & $<0.001$ \\
model 10 vs model 2 & 0.026 & 3.299 & $\mathbf{0 . 0 0 1}$ & $0.179(0.010,0.260)$ & $<0.001$ & $0.081(0.050,0.112)$ & $<0.001$ \\
model 10 vs model 7 & 0.015 & 2.907 & $\mathbf{0 . 0 0 4}$ & $0.105(0.036,0.173)$ & $\mathbf{0 . 0 0 3}$ & $0.049(0.024,0.073)$ & $<0.001$ \\
model 10 vs model 8 & 0.021 & 2.551 & $\mathbf{0 . 0 1 1}$ & $0.172(0.086,0.259)$ & $<0.001$ & $0.052(0.028,0.076)$ & $<0.001$ \\
model 10 vs model 9 & 0.026 & 3.299 & $\mathbf{0 . 0 0 1}$ & $0.179(0.098,0.260)$ & $<0.001$ & $0.081(0.050,0.112)$ & $<0.001$ \\
\hline
\end{tabular}
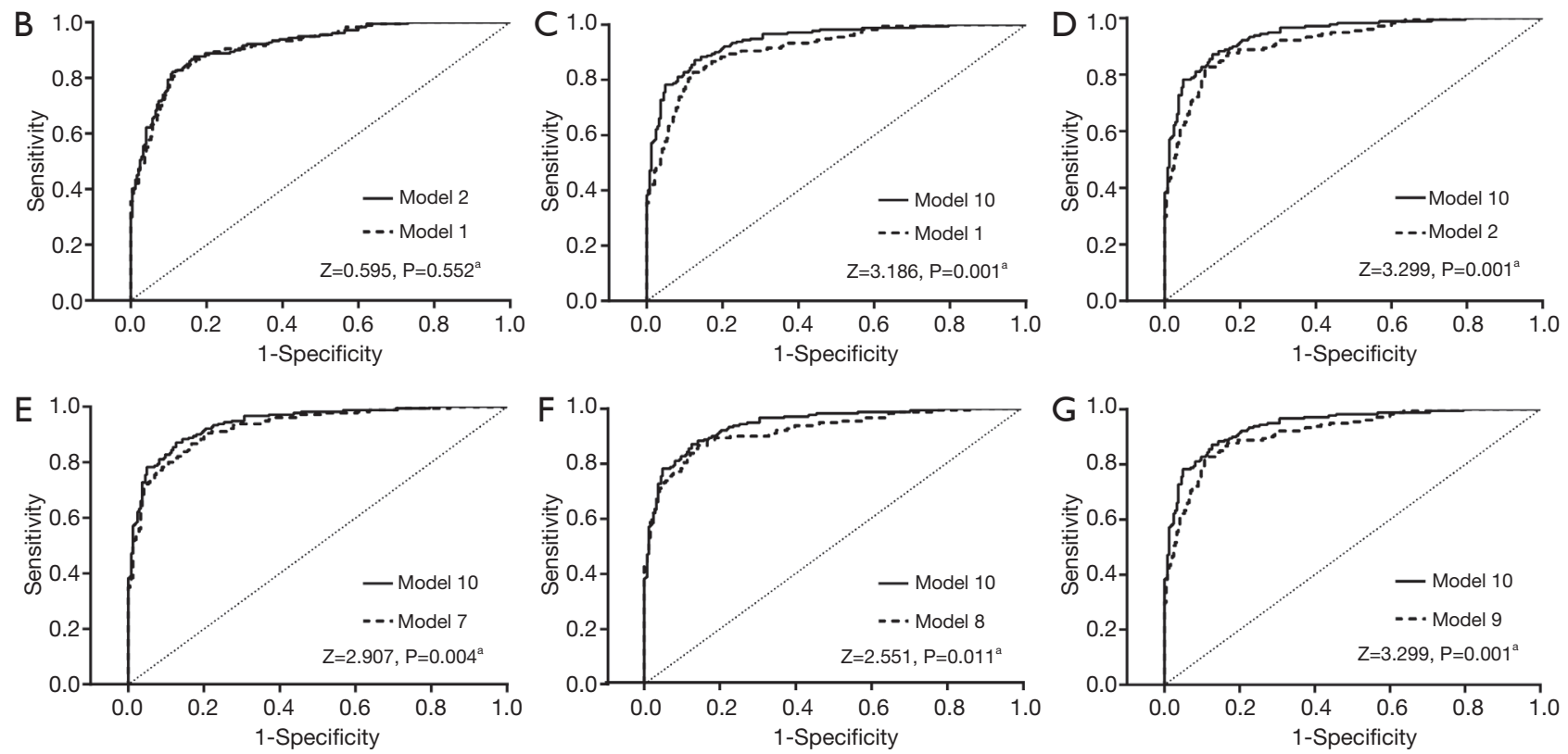

Figure 3 Comparisons of significant multivariable logistic regression models for CAD risk assessment. Model 1, model 2, and models 7 to 10 were defined as significant models with significant ORs of HDL parameters and inflammatory markers $(\mathrm{P}<0.05)$. (A) Comparisons of $Z$ value, NRI, and IDI of the six significant models. The $Z$ value represents the comparison of $C$-statistics; $\mathrm{P}$ value ${ }^{\mathrm{a}}$ represents the significance of the $\mathrm{Z}$ value. NRI, net reclassification improvement, was calculated with clinically based cut-off points for CAD risk as $10 \%$ and $20 \%$; $\mathrm{P}$ value $^{\mathrm{b}}$ for significance of NRI. IDI, integrated discrimination improvement; $\mathrm{P}$ value ${ }^{\mathrm{c}}$ for significance of IDI. (B) ROC curve for comparison of model 2 with model 1 in the C-statistic. (C,D,E,F) ROC curves for comparisons of model 10 with models 1, 2, 7, and 8 in the C-statistics. (G) ROC curve for comparison of model 10 with model 9 in the $\mathrm{C}$-statistic. $\mathrm{P}<0.05$ as significance. HDL, high-density lipoprotein; CAD, coronary artery disease; OR, odds ratio; NRI, net reclassification improvement; IDI, integrated discrimination improvement; ROC, receiver operating characteristic.

significant differences in pairwise comparisons among the L/M/H-FR and CAD groups and remained inversely associated with CAD independent of CRFs. Consistent with our findings, previous data have yielded an inverse relationship between $\mathrm{HDL}_{2}$ levels and CAD $(9,10,24)$, carotid atherosclerosis $(7,25)$, and type 2 diabetes (26). Cholesterol efflux capacity (CEC) is crucial to HDL in the anti-atherogenic process. El Khoudary et al. (27) found a strong correlation of large-HDL subspecies with macrophage CEC in healthy women before and after menopause. In patients with coronary endothelial dysfunction, an early marker of atherosclerosis, the selective decrease in large-HDL concentrations had already contributed to impaired cholesterol efflux from endothelial cells (28). However, a more protective effect of $\mathrm{HDL}_{3}$ over $\mathrm{HDL}_{2}$ (29) or equal benefits of both subfractions for CAD (30) has also been reported. Martin et al. (13) have also revealed that the central positioning and inefficiency of $\mathrm{HDL}_{3}$ in cholesterol efflux potentially explained the trend of lower $\mathrm{HDL}_{3}$ with a higher risk for mortality/ myocardial infarction in people with secondary prevention. This inconsistency may be caused by the differences in the 
health status of the populations studied, the methods used to subclassify HDL-C, and perhaps the anti-atherosclerotic property of various HDL particles.

In addition, although the model with $\mathrm{HDL}_{2}$ yielded no fit improvement over the model with HDL-C, only $\mathrm{HDL}_{2}$ showed significance when applying all six HDL parameters in the CAD risk model with adjustment for CRFs. This suggests a possible superiority of $\mathrm{HDL}_{2}$ to $\mathrm{HDL}-\mathrm{C}$ in $\mathrm{CAD}$ risk prediction. $\mathrm{HDL}_{2}$ has been proven to be more closely related to carotid intima-media thickness than total HDL-C in healthy middle-aged individuals (31). Lamon-Fava et al. (32) also found that the power of large-HDL particles quantified by $2 \mathrm{D}$ gel electrophoresis to predict coronary atherosclerosis risk was typically superior to that of HDL-C in postmenopausal women. However, some studies elucidated that neither $\mathrm{HDL}_{2}$ nor $\mathrm{HDL}_{3}$ was better at $\mathrm{CAD}$ risk prediction than HDL-C itself (33). The superiority of $\mathrm{HDL}_{2}$ over HDL-C requires verification in a large-scale prospective study.

Inflammation is ubiquitous in the atherothrombotic process, and inflammatory biomarkers, including MCP1 and hsCRP, were positively related to CAD risk independent of CRFs in our study. MCP-1 is likely to play a pathogenic role in $\mathrm{CAD}$ by recruiting monocytes to initiate plaque formation and activating the ubiquitin-proteasome system to trigger plaque rupture (34). Therefore, its levels may reflect increased atherosclerotic burden, enhanced plaque vulnerability, or both (35). We also found that MCP1 levels were similar in the L- and M-FR groups but showed a significant difference between the $M-$ and H-FR groups, indicating that MCP-1 could be applied in discriminating persons with high CAD risk among asymptomatic subjects for more aggressive intervention.

Different from MCP-1, hsCRP is predominantly a biomarker of inflammation rather than a causal factor (36). Increased hsCRP levels were associated with an increased risk of CAD and ischaemic stroke (17) and adds as much to risk prediction as total cholesterol, HDL-C and blood pressure (18). The difference in hsCRP levels was significant between the L- and M-FR groups but lost significance when comparing M-FR with the H-FR group, which could be explained by hsCRP being an acute reactant that responds easily and quickly to low-grade inflammation while maintaining a stable level in persistent inflammation (35).

The incidence of CAD is strongly associated with a set of traditional risk factors, and the inclusion of new risk factors may improve the performance of current multivariable risk assessment tools. By incorporating hsCRP and a family history of premature CAD, the Reynolds Risk Score shows improved calibration and discrimination over the Framingham cardiovascular risk score and the Adult Treatment Panel III score (37). Therefore, we applied all six HDL parameters and two inflammatory markers into the CRFs-adjusted model, in which $\mathrm{HDL}_{2}$, combined MCP1 and hsCRP, showed significance. Impressively, this model has achieved significant improvement in discrimination and risk reclassification over the models including either a single factor alone or all six HDL parameters together. CanouiPoitrine et al. (16) also added two inflammatory biomarkers, RANTES and IP-10, to the traditional risk factor-based model for ischaemic stroke risk and gained improvement in both C-statistics and reclassification. However, the combined value of HDL subfractions and inflammatory markers needs to be evaluated in further prospective studies. To an extent, our data suggest that the combined screening of HDL subfractions with inflammatory biomarkers may provide better predictive information than screening for either a biomarker alone or all six HDL parameters together.

\section{Limitations}

First, this was a cross-sectional study, and the results should be confirmed in prospective trials. Additionally, the study was conducted in a single centre with a relatively small sample size, and conflict existed regarding whether $\mathrm{HDL}_{2}$ was superior to HDL-C in CAD risk assessment. Further exploration is needed in a larger-scale study.

\section{Conclusions}

In conclusion, the large $\mathrm{HDL}_{2}$ is superior to small $\mathrm{HDL}_{3}$ in the assessment of CAD risk. In the model including all six HDL parameters, only $\mathrm{HDL}_{2}$, but not HDL-C, maintained the inverse and independent association with CAD, revealing the possible superiority of $\mathrm{HDL}_{2}$ to $\mathrm{HDL}-\mathrm{C}$. The combination of $\mathrm{HDL}_{2}, \mathrm{MCP}-1$, and hsCRP with CRFs provides an optimal and validated prediction for CAD than a single biomarker alone or all HDL parameters together.

\section{Acknowledgments}

Funding: The authors disclosed receipt of the following financial support for the research, authorship, and/ or publication of this article: This study was supported by grants from the Natural Science Foundation of 


\section{Page 10 of 11}

China (Grant number: 81970388, 81700397, 81570213, 81870170), and Natural Science Foundation of Guangdong Province (Grant number: 2019A1515011682), and Guangzhou Science and Technology Program projects (Grant number: 201803040010), and Bioland Laboratory (Guangzhou Regenerative Medicine and Health Guangdong Laboratory) (Grant number: 2019GZR110406004).

\section{Footnote}

Reporting Checklist: The authors have completed the MDAR checklist. Available at http://dx.doi.org/10.21037/atm-21948

Data Sharing Statement: Available at http://dx.doi. org/10.21037/atm-21-948

Conflicts of Interest: All authors have completed the ICMJE uniform disclosure form (available at http://dx.doi. org/10.21037/atm-21-948). The authors have no conflicts of interest to declare.

Ethical Statement: The authors are accountable for all aspects of the work in ensuring that questions related to the accuracy or integrity of any part of the work are appropriately investigated and resolved. The study complied with the Declaration of Helsinki (as revised in 2013) and was approved by the hospital's ethics review board (Sun Yat-sen Memorial Hospital, Guangzhou, China, IRB number SYSEC-KYKS-2020-083-001). Informed consent was obtained from all individual participants included in the study.

Open Access Statement: This is an Open Access article distributed in accordance with the Creative Commons Attribution-NonCommercial-NoDerivs 4.0 International License (CC BY-NC-ND 4.0), which permits the noncommercial replication and distribution of the article with the strict proviso that no changes or edits are made and the original work is properly cited (including links to both the formal publication through the relevant DOI and the license). See: https://creativecommons.org/licenses/by-nc-nd/4.0/.

\section{References}

1. Gordon T, Castelli WP, Hjortland MC, et al. High density lipoprotein as a protective factor against coronary heart disease. The Framingham Study. Am J Med 1977;62:707-14.
Wu et al. $\mathrm{HDL}_{2}$ and inflammatory factors for CAD risk prediction

2. Gordon DJ, Probstfield JL, Garrison RJ, et al. Highdensity lipoprotein cholesterol and cardiovascular disease. Four prospective American studies. Circulation 1989;79:8-15.

3. Di Angelantonio E, Sarwar N, Perry P, et al. Major lipids, apolipoproteins, and risk of vascular disease. JAMA 2009;302:1993-2000.

4. Bowman L, Hopewell JC, Chen F, et al. Effects of anacetrapib in patients with atherosclerotic vascular disease. N Engl J Med 2017;377:1217-27.

5. Schwartz GG, Olsson AG, Abt M, et al. Effects of dalcetrapib in patients with a recent acute coronary syndrome. N Engl J Med 2012;367:2089-99.

6. Oldoni F, Sinke RJ, Kuivenhoven JA. Mendelian disorders of high-density lipoprotein metabolism. Circ Res 2014;114:124-42.

7. Tiozzo E, Gardener H, Hudson BI, et al. Subfractions of high-density lipoprotein-cholesterol and carotid intimamedia thickness: the Northern Manhattan Study. Stroke 2016;47:1508-13.

8. Hirano T, Nohtomi K, Koba S, et al. A simple and precise method for measuring HDL-cholesterol subfractions by a single precipitation followed by homogenous HDLcholesterol assay. J Lipid Res 2008;49:1130-6.

9. Salonen JT, Salonen R, Seppänen K, et al. HDL, HDL2, and HDL3 subfractions, and the risk of acute myocardial infarction. A prospective population study in eastern Finnish men. Circulation 1991;84:129-39.

10. Laakso M, Lehto S, Penttilä I, et al. Lipids and lipoproteins predicting coronary heart disease mortality and morbidity in patients with non-insulin-dependent diabetes. Circulation 1993;88:1421-30.

11. Sun RL, Huang CX, Bao JL, et al. CC-chemokine ligand 2 (CCL2) suppresses high density lipoprotein (HDL) internalization and cholesterol efflux via CC-chemokine receptor 2 (CCR2) induction and p42/44 mitogenactivated protein kinase (MAPK) activation in human endothelial cells. J Biol Chem 2016;291:19532-44.

12. Sweetnam PM, Bolton CH, Yarnell JW, et al. Associations of the HDL2 and HDL3 cholesterol subfractions with the development of ischemic heart disease in British men. The Caerphilly and speedwell collaborative heart disease studies. Circulation 1994;90:769-74.

13. Martin SS, Khokhar AA, May HT, et al. HDL cholesterol subclasses, myocardial infarction, and mortality in secondary prevention: the lipoprotein investigators collaborative. Eur Heart J 2015;36:22-30.

14. Geovanini GR, Libby P. Atherosclerosis and inflammation: 
overview and updates. Clin Sci (Lond) 2018;132:1243-52.

15. Georgakis MK, Gill D, Rannikmae K, et al. Genetically determined levels of circulating cytokines and risk of stroke. Circulation 2019;139:256-68.

16. Canouï-Poitrine F, Luc G, Mallat Z, et al. Systemic chemokine levels, coronary heart disease, and ischemic stroke events: the PRIME study. Neurology 2011;77:1165-73.

17. Kaptoge S, Di Angelantonio E, Pennells L, et al. C-reactive protein, fibrinogen, and cardiovascular disease prediction. N Engl J Med 2012;367:1310-20.

18. Kaptoge S, Di Angelantonio E, Lowe G, et al. C-reactive protein concentration and risk of coronary heart disease, stroke, and mortality: an individual participant metaanalysis. Lancet 2010;375:132-40.

19. Lin JS, Evans CV, Johnson E, et al. Nontraditional risk factors in cardiovascular disease risk assessment: updated evidence report and systematic review for the US preventive services task force. JAMA 2018;320:281-97.

20. Wilson PW, D'Agostino RB, Levy D, et al. Prediction of coronary heart disease using risk factor categories. Circulation 1998;97:1837-47.

21. D'Agostino RB, Sr., Vasan RS, Pencina MJ, et al. General cardiovascular risk profile for use in primary care: the Framingham Heart Study. Circulation 2008;117:743-53.

22. DeFilippis AP, Young R, Carrubba CJ, et al. An analysis of calibration and discrimination among multiple cardiovascular risk scores in a modern multiethnic cohort. Ann Intern Med 2015;162:266-75.

23. Ridker PM, Rifai N, Rose L, et al. Comparison of C-reactive protein and low-density lipoprotein cholesterol levels in the prediction of first cardiovascular events. $\mathrm{N}$ Engl J Med 2002;347:1557-65.

24. Morgan J, Carey C, Lincoff A, et al. High-density lipoprotein subfractions and risk of coronary artery disease. Curr Atheroscler Rep 2004;6:359-65.

25. Alagona C, Soro A, Ylitalo K, et al. A low high density lipoprotein (HDL) level is associated with carotid artery intima-media thickness in asymptomatic members of low HDL families. Atherosclerosis 2002;165:309-16.

26. Tabara Y, Arai H, Hirao Y, et al. Different inverse association of large high-density lipoprotein subclasses with exacerbation of insulin resistance and incidence of type 2 diabetes: the Nagahama study. Diabetes Res Clin Pract 2017;127:123-31.

27. El Khoudary SR, Hutchins PM, Matthews KA, et al. Cholesterol efflux capacity and subclasses of HDL particles in healthy women transitioning through menopause. J Clin Endocrinol Metab 2016;101:3419-28.
28. Monette JS, Hutchins PM, Ronsein GE, et al. Patients with coronary endothelial dysfunction have impaired cholesterol efflux capacity and reduced HDL particle concentration. Circ Res 2016;119:83-90.

29. Joshi PH, Toth PP, Lirette ST, et al. Association of highdensity lipoprotein subclasses and incident coronary heart disease: the Jackson Heart and Framingham Offspring Cohort Studies. Eur J Prev Cardiol 2016;23:41-9.

30. Buring JE, O'Connor GT, Goldhaber SZ, et al. Decreased HDL2 and HDL3 cholesterol, Apo A-I and Apo A-II, and increased risk of myocardial infarction. Circulation 1992;85:22-9.

31. Föger B, Luef G, Ritsch A, et al. Relationship of highdensity lipoprotein subfractions and cholesteryl ester transfer protein in plasma to carotid artery wall thickness. J Mol Med (Berl) 1995;73:369-72.

32. Lamon-Fava S, Herrington DM, Reboussin DM, et al. Plasma levels of HDL subpopulations and remnant lipoproteins predict the extent of angiographicallydefined coronary artery disease in postmenopausal women. Arterioscler Thromb Vasc Biol 2008;28:575-9.

33. Rader DJ, Hovingh GK. HDL and cardiovascular disease. Lancet 2014;384:618-25.

34. Marfella R, D'Amico M, Di Filippo C, et al. Increased activity of the ubiquitin-proteasome system in patients with symptomatic carotid disease is associated with enhanced inflammation and may destabilize the atherosclerotic plaque: effects of rosiglitazone treatment. J Am Coll Cardiol 2006;47:2444-55.

35. de Lemos JA, Morrow DA, Blazing MA, et al. Serial measurement of monocyte chemoattractant protein-1 after acute coronary syndromes: results from the A to $Z$ trial. J Am Coll Cardiol 2007;50:2117-24.

36. Ridker PM. A test in context: high-sensitivity C-reactive protein. J Am Coll Cardiol 2016;67:712-23.

37. Cook NR, Paynter NP, Eaton CB, et al. Comparison of the Framingham and Reynolds risk scores for global cardiovascular risk prediction in the multiethnic Women's Health Initiative. Circulation 2012;125:1748-56.

(English Language Editor: B. Draper)

Cite this article as: $\mathrm{Wu} \mathrm{X}, \mathrm{He} \mathrm{Z}$, Sun $\mathrm{R}$, Xie $\mathrm{X}$, Chen $\mathrm{Q}$, Wang J, Bao J, Huang J, Jiang Y, Zhang Y, Wang J. Large $\mathrm{HDL}_{2}$ combined with inflammatory factors as superior predictors for coronary artery disease than small $\mathrm{HDL}_{3}$. Ann Transl Med 2021;9(8):672. doi: 10.21037/atm-21-948 